\title{
New Allyldithiocarbimates: Synthesis, Structure and Antifungal Activity against Phakopsora pachyrhizi and Hemileia vastatrix
}

\author{
Antonio E. C. Vidigal, ${ }^{a}$ Mayura M. M. Rubinger, ${ }^{\circledR *, a}$ Lucas F. da Silva, ${ }^{b}$ \\ Laércio Zambolim, ${ }^{b}$ Arthur B. D. Pereira, ${ }^{a}$ Silvana Guilardi, ${ }^{c}$ Rafael A. C. Souza ${ }^{c}$ \\ and Javier Ellena ${ }^{d}$
}

${ }^{a}$ Departamento de Química, Universidade Federal de Viçosa, 36570-977 Viçosa-MG, Brazil

${ }^{b}$ Departamento de Fitopatologia, Universidade Federal de Viçosa, 36570-977 Viçosa-MG, Brazil

'Instituto de Química, Universidade Federal de Uberlândia, 38400-902 Uberlândia-MG, Brazil

${ }^{d}$ Instituto de Física de São Carlos, Universidade de São Paulo, 13566-590 São Carlos-SP, Brazil

Twelve tetraphenylphosphonium allyldithiocarbimates were synthesized and fully characterized by high-resolution electrospray ionization mass spectrometry (HR-ESI-MS), infrared and nuclear magnetic resonance (NMR) spectroscopies. The spectroscopic data indicated that the allyldithiocarbimate anions present $Z$ configuration, as confirmed by X-ray crystallography. These new compounds inhibited the germination of Phakopsora pachyrhizi and Hemileia vastatrix at very low doses, with $\mathrm{IC}_{50}$ (concentration to achieve $50 \%$ of inhibition of spore germination) values ranging from 0.028 to $0.166 \mathrm{mmol} \mathrm{L}^{-1}$, and $\mathrm{IC}_{90}$ (concentration to achieve $90 \%$ of inhibition of spore germination) values varying from 0.156 to $0.528 \mathrm{mmol} \mathrm{L}^{-1}$. Allyldithiocarbimate salts are lead compounds for the development of new antifungals for coffee leaf rust and Asian soybean rust, devastating plant diseases with limited control options.

Keywords: allyldithiocarbimate, crystal structure, antifungal activity, Hemileia vastatrix, Phakopsora pachyrhizi

\section{Introduction}

Dithiocarbamates (Figure 1) are protectant fungicides extensively used in agriculture. For example, Ziram, whose active principle is the zinc dimethyldithiocarbamate (ZDMC), is used either single or in mixtures with more specific systemic fungicides. ${ }^{1}$ Even though the metaldithiocarbamates do not present high toxicity when compared to other fungicides, the literature ${ }^{2}$ reports that Ziram and other zinc-dithiocarbamates increase the<smiles>[R]N([R])C(=S)[S-]</smiles>

(a)

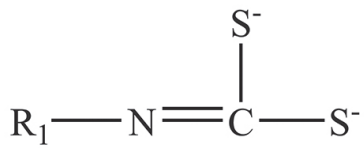

(b)
Figure 1. General formulae of dithiocarbamate (a) and dithiocarbimate (b) anions.

\footnotetext{
*e-mail: mayura@ufv.br
}

intracellular levels of $\mathrm{Zn}^{2+}$, which might induce apoptosis and other adverse events. In addition, it has been recently discovered that Ziram inhibits androgen production and steroidogenic enzyme activities in rat Leydig cells, in vitro. ${ }^{3}$

Although similar to the dithiocarbamates, the dithiocarbimates (Figure 1) are much less studied and have no commercial applications yet. In previous research, it was found that $N$-R-sulfonyldithiocarbimate metal complexes (metals: $\mathrm{Zn}, \mathrm{Ni}, \mathrm{Sn}$ ) inhibit the mycelial growth of Colletotrichum, Botrytis and Alternaria spp., in vitro. ${ }^{4-7}$ The potassium salts of the free dithiocarbimate ligands are not stable enough in solution for accurate biological studies. ${ }^{4}$ Attempting to synthesize stable metal-free organic dithiocarbimates derivatives, we have recently prepared the first examples of allyldithiocarbimates, via nucleophilic substitution reactions of aromatic sulfonyldithiocarbimates with Morita-Baylis-Hillman (MBH) adducts derivatives. These compounds were also active, inhibiting the mycelial growth of B. cinerea, in vitro. ${ }^{8}$

Considering the potential application of this new class of substances as agrochemicals, here we present 
an extension of this research, with the syntheses of twelve new allyldithiocarbimate salts, now derived from aliphatic sulfonyldithiocarbimates and different MBH derivatives. The allyldithiocarbimate anions were isolated as tetraphenylphosphonium salts and were fully characterized by high-resolution electrospray ionization mass spectrometry (HR-ESI-MS), infrared and nuclear magnetic resonance (NMR) spectroscopies. $\mathrm{X}$-ray diffraction experiments supported the proposed structures.

This work also presents a study on the activities of the allyldithiocarbimate salts against Hemileia vastatrix and Phakopsora pachyrhizi. Severe coffee leaf-rust epidemics have affected a number of countries, especially from Peru to Mexico, in the last decade, being the most important disease in the Brazilian coffee crops. The causal agent, $H$. vastatrix, is an obligate parasite and affects the aerial part of the plant, causing an early fall of leaves and drying of the branches, consequently reducing productivity. ${ }^{9,10}$ Asian soybean rust, caused by P. pachyrhizi is also a major problem in Brazil. Original from Asia, the fungus was first detected in 2001 in Paraguay, spreading within four years throughout the continent, up to the United States of America. Loss of sensibility of the fungus to available fungicides has already been reported. ${ }^{11}$

\section{Experimental}

\section{Methods and materials}

Melting points (mp) were determined with an MQAPF-302 equipment (Microquímica) and are reported without correction. High resolution mass spectra (HRMS) were recorded on a micrOTOF Q-II liquid chromatography mass spectrometer (UltraflexIII, Brucker Daltonics) under electrospray ionization (ESI). Infrared (IR) spectra were recorded on a Varian 660-IR, equipped with GladiATR (attenuated total reflection, ATR) scanning from 4000 to $500 \mathrm{~cm}^{-1}$. The ${ }^{1} \mathrm{H}$ and ${ }^{13} \mathrm{C}$ NMR spectra were recorded on a Varian Mercury 300 instrument (300 and $75 \mathrm{MHz}$, respectively), using deuterated chloroform as solvent and tetramethylsilane (TMS) as internal standard. Nuclear Overhauser effect spectroscopy (NOESY) experiments were performed for structural characterization of the reaction products. Thin layer chromatography (TLC) analysis was conducted on aluminum precoated silica gel plates. Carbon disulfide, potassium hydroxide, ammonia aqueous solution, dimethyl sulfoxide and dimethylformamide were purchased from Vetec (Duque de Caxias, Brazil). The remaining reagents were purchased from Sigma-Aldrich (St. Louis, USA) and were used without further purification.

\section{Syntheses of the precursors}

The potassium $N$-R-sulfonyldithiocarbimates were prepared by the reaction of the appropriated sulfonamides with carbon disulfide and two molar equivalents of potassium hydroxide in dimethylformamide (Scheme 1), as described in the literature. ${ }^{12-14}$ The potassium dithiocarbimates were characterized by infrared and NMR spectroscopies, in comparison with reported data. ${ }^{12-14}$

$$
\begin{gathered}
\mathrm{R}-\mathrm{SO}_{2} \mathrm{NH}_{2} \underset{\text { dimethylformamide }}{\stackrel{\mathrm{CS}_{2}, \mathrm{KOH}}{\longrightarrow}} \mathrm{K}_{2}\left(\mathrm{R}-\mathrm{SO}_{2} \mathrm{~N}=\mathrm{CS}_{2}\right) \\
\mathrm{R}=\mathrm{CH}_{3}, \mathrm{C}_{2} \mathrm{H}_{5}, \mathrm{C}_{4} \mathrm{H}_{9}, \mathrm{C}_{8} \mathrm{H}_{17}
\end{gathered}
$$

Scheme 1. Preparation of potassium $N$-R-sulfonyldithiocarbimates.

Three MBH adducts (Scheme 2) were prepared by the reaction of methyl acrylate with benzaldehyde, 4-nitrobenzaldehyde or 4-(trifluoromethyl)benzaldehyde, catalyzed by trimethylamine, ${ }^{15}$ and were converted into the respective allylic bromides (Scheme 2), in reaction with lithium bromide and sulfuric acid, as described in the literature. ${ }^{16,17}$ The allylic bromides were characterized by infrared and NMR spectroscopies, in comparison with reported data. ${ }^{16,17}$

\section{Syntheses of the allyldithiocarbimates (1a-d, 2a-d and 3a-d)}

A solution of $1 \mathrm{mmol}$ of each allylic bromide in acetone $(2 \mathrm{~mL})$ was added dropwise to a stirring acetone:water (1:1 by volume) solution ( $10 \mathrm{~mL}$ ) containing $1.2 \mathrm{mmol}$ of the appropriate potassium $N$-R-sulfonyldithiocarbimate (Scheme 3). The mixture was stirred for up to $15 \mathrm{~min}$ (monitored by TLC) at room temperature. Then, water

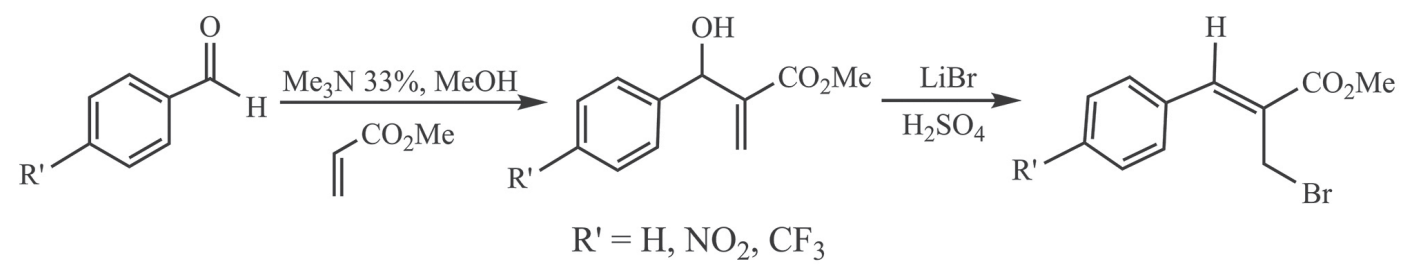

Scheme 2. Preparation of the Morita-Baylis-Hillman derivatives. 


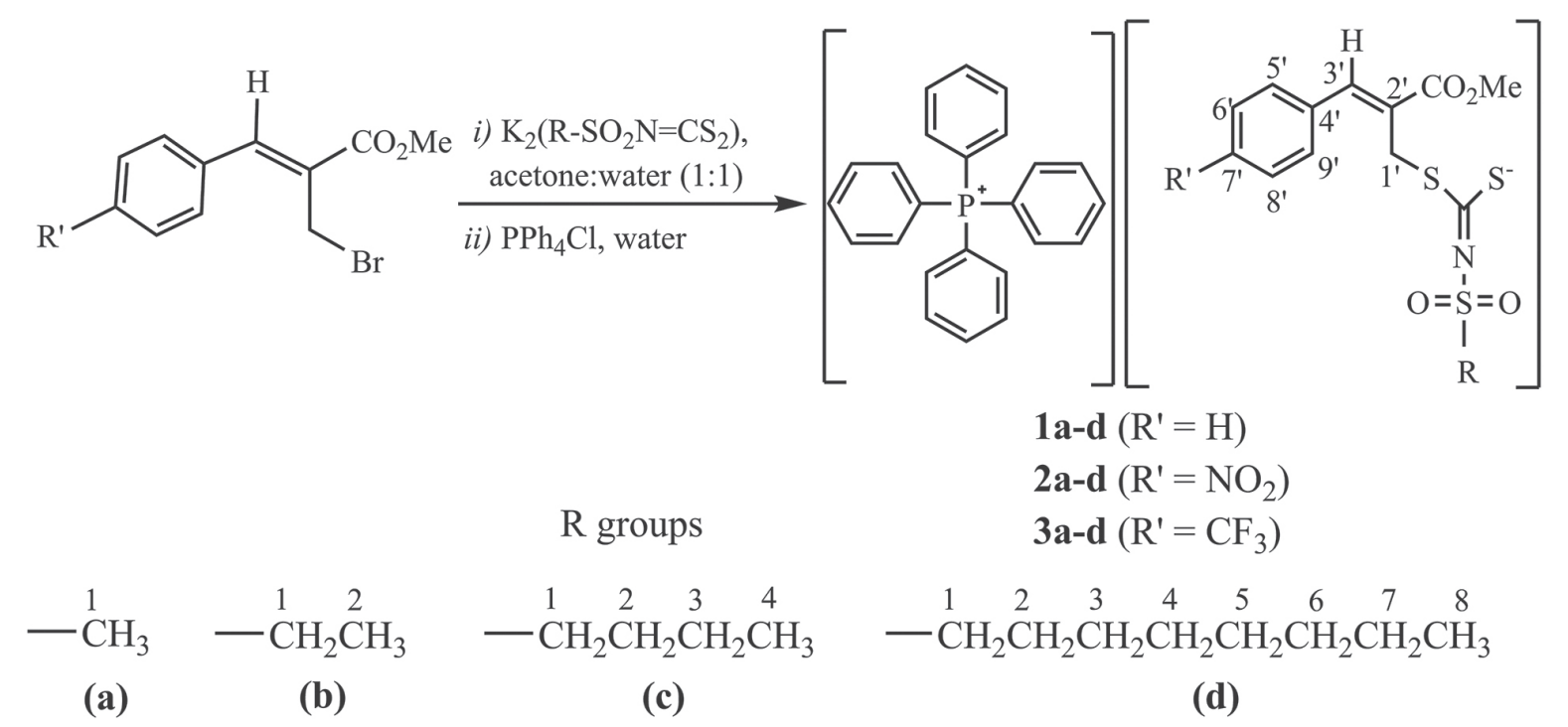

Scheme 3. Syntheses of the allyldithiocarbimates and numbering for NMR attribution.

$(5 \mathrm{~mL})$ was added and the product was extracted with ethyl acetate $(3 \times 20 \mathrm{~mL})$.

For the syntheses of compounds 1a-c, 2a-c and 3a-c, the organic phase was concentrated under reduced pressure and the residue was dissolved in water. Tetraphenylphosphonium chloride ( $1 \mathrm{mmol}$ ) was added and the mixture was stirred for $5 \mathrm{~min}$. The yellow solid thus formed was filtered, washed with distilled water and dried under reduced pressure for one day. As compounds $\mathbf{1 d}, \mathbf{2} \mathbf{d}$ and $\mathbf{3 d}$ are oils, in these cases tetraphenylphosphonium chloride $(1 \mathrm{mmol})$ was added directly to the organic phase (ethyl acetate). The mixture was stirred for $5 \mathrm{~min}$. Then the organic solution was washed with water $(2 \times 20 \mathrm{~mL})$, the organic solvent was evaporated, and the residue was dried under reduced pressure for one day.

The HRMS spectra of the products (1a-d, 2a-d, 3a-d) presented one peak at $\mathrm{m} / \mathrm{z} 339.1318$, in the positive mode, due to the tetraphenylphosphonium cation. Their NMR spectra showed the expected signals for the tetraphenylphosphonium cation at: ${ }^{1} \mathrm{H}$ NMR $(300 \mathrm{MHz}$, $\left.\mathrm{CDCl}_{3}\right) \delta$ 7.50-7.68 $\left(\mathrm{m}^{*}, 8 \mathrm{H}\right), 7.70-7.81\left(\mathrm{~m}^{*}, 8 \mathrm{H}\right)$, 7.85-7.93 (m, 4H) (*superimposed on the signals of H3', $\mathrm{H} 5$ ' and $\mathrm{H} 9{ }^{\prime}$ of the anions); ${ }^{13} \mathrm{C} \mathrm{NMR}\left(75 \mathrm{MHz}, \mathrm{CDCl}_{3}\right.$ ) $\delta 117.5\left(\mathrm{~d},{ }^{1} J_{\mathrm{C}-\mathrm{P}} 90 \mathrm{~Hz}\right), 130.8\left(\mathrm{~d},{ }^{2} J_{\mathrm{C}-\mathrm{P}} 7.5 \mathrm{~Hz}\right), 134.4(\mathrm{~d}$, $\left.{ }^{3} J_{\mathrm{C}-\mathrm{P}} 7.5 \mathrm{~Hz}\right), 135.8\left(\mathrm{~d},{ }^{4} J_{\mathrm{C}-\mathrm{P}} 3.0 \mathrm{~Hz}\right)$. The remaining signals and other experimental data are as follows.

Tetraphenylphosphonium (Z)-2-(methoxycarbonyl)3-phenylallyl-( $N$-methylsulfonyl)dithiocarbimate (1a)

Yield 80\%; mp 117.3-118.9 ${ }^{\circ} \mathrm{C}$; IR (selected bands, ATR $v / \mathrm{cm}^{-1} 1697(v \mathrm{C}=\mathrm{O}), 1373(v \mathrm{C}=\mathrm{N}), 1269\left(v_{\mathrm{as}} \mathrm{SO}_{2}\right)$, $1140\left(v_{\text {sym }} \mathrm{SO}_{2}\right), 924\left(v_{\mathrm{as}} \mathrm{CS}_{2}\right) ;{ }^{1} \mathrm{H}$ NMR (allyldithiocarbimate signals, $\left.300 \mathrm{MHz}, \mathrm{CDCl}_{3}\right) \delta 3.23(\mathrm{~s}, 3 \mathrm{H}, \mathrm{H1}), 3.72(\mathrm{~s}$, $\left.3 \mathrm{H}, \mathrm{OCH}_{3}\right), 4.20$ (s, 2H, H1'), 7.22-7.37 (m, 3H, H6', H7', H8'), 7.49-7.97 (m*, 3H, H3', H5', H9'); ${ }^{13} \mathrm{C}$ NMR (allyldithiocarbimate signals, $75 \mathrm{MHz}, \mathrm{CDCl}_{3}$ ) $\delta 33.3$ (C1'), $39.0(\mathrm{C} 1), 52.1\left(\mathrm{OCH}_{3}\right), 127.3\left(\mathrm{C} 2^{\prime}\right), 128.6$ (C6' and C8'), 128.8 (C7'), 130.1 (C5' and C9'), 134.8 (C4'), 141.3 (C3'), $168.3(\mathrm{C}=\mathrm{O}), 201.6(\mathrm{C}=\mathrm{N})$; HRMS (ESI) $\mathrm{m} / z$, calcd. for $\mathrm{C}_{13} \mathrm{H}_{14} \mathrm{NO}_{4} \mathrm{~S}_{3}^{-}: 344.0090$, found: 344.0107 .

Tetraphenylphosphonium (Z)-2-(methoxycarbonyl)3-phenylallyl-( $N$-ethylsulfonyl)dithiocarbimate (1b)

Yield 82\%; mp 114.5-116.2 ${ }^{\circ} \mathrm{C}$; IR (selected bands, ATR $) v / \mathrm{cm}^{-1} 1697(v \mathrm{C}=\mathrm{O}), 1365(v \mathrm{C}=\mathrm{N}), 1257\left(v_{\mathrm{as}} \mathrm{SO}_{2}\right)$, $1142\left(v_{\mathrm{sym}} \mathrm{SO}_{2}\right), 930\left(v_{\mathrm{as}} \mathrm{CS}_{2}\right) ;{ }^{1} \mathrm{H} \mathrm{NMR}$ (allyldithiocarbimate signals, $\left.300 \mathrm{MHz}, \mathrm{CDCl}_{3}\right) \delta 1.28$ (t, $3 \mathrm{H}, J 7.5 \mathrm{~Hz}, \mathrm{H} 2$ ), 3.46 (q, 2H, J 7.5 Hz, H1), 3.74 (s, 3H, $\mathrm{OCH}_{3}$ ), 4.18 (s, 2H, H1'), 7.27-7.37 (m, 3H, H6', H7', H8'), 7.47-7.95 (m*, 3H, H3', H5', H9'); ${ }^{13} \mathrm{C}$ NMR (allyldithiocarbimate signals, $\left.75 \mathrm{MHz}, \mathrm{CDCl}_{3}\right) \delta 8.4\left(\mathrm{C}^{2}\right), 33.4\left(\mathrm{Cl}^{\prime}\right.$ ) $), 45.8(\mathrm{C} 1)$, $52.3\left(\mathrm{OCH}_{3}\right), 127.3\left(\mathrm{C}^{\prime}{ }^{\prime}\right), 128.6\left(\mathrm{C} 6^{\prime}\right.$ and $\left.\mathrm{C} 8^{\prime}\right), 128.9\left(\mathrm{C}^{\prime}\right)$, 130.1 (C5' and C9'), 134.8 (C4'), 141.5 (C3'), $168.3(\mathrm{C}=\mathrm{O})$, $201.6(\mathrm{C}=\mathrm{N})$; HRMS (ESI) $\mathrm{m} / z$, calcd. for $\mathrm{C}_{14} \mathrm{H}_{16} \mathrm{NO}_{4} \mathrm{~S}_{3}^{-}$: 358.0247, found: 358.0240 .

Tetraphenylphosphonium (Z)-2-(methoxycarbonyl)3-phenylallyl-( $N$-butylsulfonyl)dithiocarbimate (1c)

Yield 75\%; mp 91.6-92.4 ${ }^{\circ} \mathrm{C}$; IR (selected bands, ATR) $v / \mathrm{cm}^{-1} 1705(v \mathrm{C}=\mathrm{O}), 1381(v \mathrm{C}=\mathrm{N}), 1261\left(v_{\text {as }} \mathrm{SO}_{2}\right), 1147$ $\left(v_{\text {sym }} \mathrm{SO}_{2}\right), 933\left(v_{\text {as }} \mathrm{CS}_{2}\right) ;{ }^{1} \mathrm{H}$ NMR (allyldithiocarbimate signals, $\left.300 \mathrm{MHz}, \mathrm{CDCl}_{3}\right) \delta 0.83(\mathrm{t}, 3 \mathrm{H}, J 7.5 \mathrm{~Hz}, \mathrm{H} 4)$, 1.34 (sext, 2H, J 7.5 Hz, H3), 1.70-1.82 (m, 2H, H2), 3.51 (t, $2 \mathrm{H}, J 7.9 \mathrm{~Hz}, \mathrm{H} 1), 3.69$ (s, 3H, $\left.\mathrm{OCH}_{3}\right), 4.15(\mathrm{~s}, 2 \mathrm{H}$, H1'), 7.18-7.30 (m, 3H, H6', H7', H8'), 7.50-7.68 (m*, 3H, H3', H5', H9'), 7.47-7.63 (m, 3H, H3', H5', H9'); 
${ }^{13} \mathrm{C}$ NMR (allyldithiocarbimate signals, $75 \mathrm{MHz}, \mathrm{CDCl}_{3}$ ) $\delta 13.9$ (C4), 22.0 (C3), 25.7 (C2), 33.3 (C1'), 50.9 (C1), $52.1\left(\mathrm{OCH}_{3}\right), 127.3\left(\mathrm{C}^{\prime}{ }^{\prime}\right), 128.6\left(\mathrm{C}^{\prime}{ }^{\prime}\right.$ and $\left.\mathrm{C}^{\prime}\right), 128.8\left(\mathrm{C}^{\prime}{ }^{\prime}\right)$, 130.2 (C5' and C9'), 134.8 (C4'), 141.3 (C3'), 168.3 (C=O), $200.8(\mathrm{C}=\mathrm{N})$; HRMS (ESI) $\mathrm{m} / z$, calcd. for $\mathrm{C}_{16} \mathrm{H}_{20} \mathrm{NO}_{4} \mathrm{~S}_{3}{ }^{-}$: 386.0560, found: 386.0564 .

Tetraphenylphosphonium (Z)-2-(methoxycarbonyl)3-phenylallyl-( $N$-octylsulfonyl)dithiocarbimate (1d)

Yield 72\%; oil at room temperature; IR (selected bands, ATR $) v / \mathrm{cm}^{-1} 1705(v \mathrm{C}=\mathrm{O}), 1381(v \mathrm{C}=\mathrm{N}), 1259\left(v_{\mathrm{as}} \mathrm{SO}_{2}\right)$, $1147\left(v_{\text {sym }} \mathrm{SO}_{2}\right), 933\left(v_{\text {as }} \mathrm{CS}_{2}\right) ;{ }^{1} \mathrm{H}$ NMR (allyldithiocarbimate signals, $\left.300 \mathrm{MHz}, \mathrm{CDCl}_{3}\right) \delta 0.78-0.88(\mathrm{~m}, 3 \mathrm{H}, \mathrm{H} 8)$, 1.17-1.42 (m, 10H, H3-H7), 1.74-1.89 (m, 2H, H2), 3.53 (t, $2 \mathrm{H}, J 8.1 \mathrm{~Hz}, \mathrm{H} 1), 3.72$ (s, 3H, $\left.\mathrm{OCH}_{3}\right), 4.19$ (s, 2H, H1'), 7.19-7.35 (m, 3H, H6', H7', H8'), 7.50-7.68 (m*, 3H, H3', $\mathrm{H} 5$ ', H9'); ${ }^{13} \mathrm{C}$ NMR (allyldithiocarbimate signals, $75 \mathrm{MHz}$, $\mathrm{CDCl}_{3}$ ) $\delta 14.2$ (C8), 22.7 (C7), 23.7 (C6), 28.9 (C5), 29.2 (C4), 29.4 (C3), 31.9 (C2), 33.4 (C1'), 51.3 (C1), 52.1 $\left(\mathrm{OCH}_{3}\right), 127.4\left(\mathrm{C}^{\prime}{ }^{\prime}\right), 128.6$ (C6' and $\left.\mathrm{C}^{\prime}{ }^{\prime}\right), 128.8\left(\mathrm{C}^{\prime}{ }^{\prime}\right)$, 130.2 (C5' and C9'), 134.9 (C4'), 141.2 (C3'), $168.3(\mathrm{C}=\mathrm{O})$, $200.8(\mathrm{C}=\mathrm{N})$; HRMS (ESI) $\mathrm{m} / z$, calcd. for $\mathrm{C}_{20} \mathrm{H}_{28} \mathrm{NO}_{4} \mathrm{~S}_{3}{ }^{-}$: 442.1186, found: 442.1106 .

Tetraphenylphosphonium (Z)-2-(methoxycarbonyl)3-(4-nitrophenyl)allyl-( $N$-methylsulfonyl)dithiocarbimate (2a)

Yield 78\%; mp 147.8-149.5 ${ }^{\circ} \mathrm{C}$; IR (selected bands, ATR $v / \mathrm{cm}^{-1} 1720(v \mathrm{C}=\mathrm{O}), 1510\left(v_{\mathrm{as}} \mathrm{NO}_{2}\right), 1440\left(v_{\mathrm{sym}} \mathrm{NO}_{2}\right)$, $1388(v \mathrm{C}=\mathrm{N}), 1262\left(v_{\mathrm{as}} \mathrm{SO}_{2}\right), 1156\left(v_{\mathrm{sym}} \mathrm{SO}_{2}\right), 940\left(v_{\mathrm{as}} \mathrm{CS}_{2}\right)$; ${ }^{1} \mathrm{H}$ NMR (allyldithiocarbimate signals, $300 \mathrm{MHz}, \mathrm{CDCl}_{3}$ ) $\delta 3.25(\mathrm{~s}, 3 \mathrm{H}, \mathrm{H} 1), 3.79\left(\mathrm{~s}, 3 \mathrm{H}, \mathrm{OCH}_{3}\right), 4.21(\mathrm{~s}, 2 \mathrm{H}$, $\left.\mathrm{H} 1^{\prime}\right)$, 7.69-7.71 (m*, 1H, H3'), 7.73-7.84 (m*, 2H, H5', H9'), 8.15 (d, 2H, J 8.1 Hz, H6', H8'); ${ }^{13} \mathrm{C}^{\prime} \mathrm{NMR}$ (allyldithiocarbimate signals, $75 \mathrm{MHz}, \mathrm{CDCl}_{3}$ ) $\delta 32.9$ (C1'), $38.9(\mathrm{C} 1), 52.4\left(\mathrm{OCH}_{3}\right), 123.7$ (C6' and $\mathrm{C}^{\prime}$ ') 131.5 (C3'), 132.0 (C2'), 137.9 (C5' and C9'), 141.4 (C4'), 147.3 (C7'), $167.6(\mathrm{C}=\mathrm{O}), 200.7$ (C=N); HRMS (ESI) $\mathrm{m} / \mathrm{z}$, calcd. for $\mathrm{C}_{13} \mathrm{H}_{13} \mathrm{~N}_{2} \mathrm{O}_{6} \mathrm{~S}_{3}^{-}: 388.9941$, found: 388.9944 .

Tetraphenylphosphonium (Z)-2-(methoxycarbonyl)3-(4-nitrophenyl)allyl-( $N$-ethylsulfonyl)dithiocarbimate (2b)

Yield $84 \%$; mp 127.8-129.2 ${ }^{\circ} \mathrm{C}$; IR (selected bands, ATR $v / \mathrm{cm}^{-1} 1711(v \mathrm{C}=\mathrm{O}), 1518\left(v_{\mathrm{as}} \mathrm{NO}_{2}\right), 1381(v \mathrm{C}=\mathrm{N})$, $1338\left(v_{\text {sym }} \mathrm{NO}_{2}\right), 1259\left(v_{\mathrm{as}} \mathrm{SO}_{2}\right), 1144\left(v_{\mathrm{sym}} \mathrm{SO}_{2}\right), 931\left(v_{\mathrm{as}} \mathrm{CS}_{2}\right)$; ${ }^{1} \mathrm{H}$ NMR (allyldithiocarbimate signals, $300 \mathrm{MHz}, \mathrm{CDCl}_{3}$ ) $\delta 1.26$ (t, 3H, J 7.5 Hz, H2), 3.53 (q, 2H, J 7.5 Hz, H1), $3.75\left(\mathrm{~s}, 3 \mathrm{H}, \mathrm{OCH}_{3}\right), 4.18$ (s, 2H, H1'), 7.54-7.64 (m*, $1 \mathrm{H}$, H3'), 7.69-7.83 (m*, 2H, H5', H9'), 8.12 (d, $2 \mathrm{H}, J 8.7 \mathrm{~Hz}$, H6', H8'); ${ }^{13} \mathrm{C}$ NMR (allyldithiocarbimate signals, $75 \mathrm{MHz}$, $\left.\mathrm{CDCl}_{3}\right) \delta 8.4(\mathrm{C} 2), 33.0\left(\mathrm{Cl}^{\prime}\right), 45.3(\mathrm{C} 1), 52.4\left(\mathrm{OCH}_{3}\right)$, 123.7 (C6' and C8'), 130.9 (C3'), 131.5 (C2'), 137.8
(C5' and C9'), 141.4 (C4'), 147.3 (C7'), 167.6 (C=O), $200.1(\mathrm{C}=\mathrm{N})$; HRMS (ESI) $\mathrm{m} / z$, calcd. for $\mathrm{C}_{14} \mathrm{H}_{15} \mathrm{~N}_{2} \mathrm{O}_{6} \mathrm{~S}_{3}{ }^{-}$: 403.0098, found: 403.0043.

Tetraphenylphosphonium (Z)-2-(methoxycarbonyl)3-(4-nitrophenyl)allyl-( $\mathrm{N}$-butylsulfonyl)dithiocarbimate (2c)

Yield $80 \%$; mp $120.7-122.6{ }^{\circ} \mathrm{C}$; IR (selected bands, ATR $v / \mathrm{cm}^{-1} 1703(v \mathrm{C}=\mathrm{O}), 1512\left(v_{\mathrm{as}} \mathrm{NO}_{2}\right), 1392(v \mathrm{C}=\mathrm{N})$, $1342\left(v_{\text {sym }} \mathrm{NO}_{2}\right), 1255\left(v_{\mathrm{as}} \mathrm{SO}_{2}\right), 1146\left(v_{\text {sym }} \mathrm{SO}_{2}\right), 928\left(v_{\mathrm{as}} \mathrm{CS}_{2}\right)$; ${ }^{1} \mathrm{H}$ NMR (allyldithiocarbimate signals, $300 \mathrm{MHz}, \mathrm{CDCl}_{3}$ ) $\delta 0.87$ (t, 3H, J 7.5 Hz, H4), 1.38 (sext, 2H, J 7.5 Hz, H3), 1.73-1.84 (m, 2H, H2), 3.56 (t, 2H, J 7.8 Hz, H1), 3.78 (s, $\left.3 \mathrm{H}, \mathrm{OCH}_{3}\right), 4.21$ (s, 2H, H1'), 7.57-7.69 (m*, 1H, H3'), 7.72-7.83 (m*, 2H, H5', H9'), 8.15 (d, 2H, J 8.7 Hz, H6', $\mathrm{H} 8$ '); ${ }^{13} \mathrm{C}$ NMR (allyldithiocarbimate signals, $75 \mathrm{MHz}$, $\left.\mathrm{CDCl}_{3}\right) \delta 13.9(\mathrm{C} 4), 22.0(\mathrm{C} 3), 25.8(\mathrm{C} 2), 33.0\left(\mathrm{C}^{\prime}{ }^{\prime}\right)$, $50.9(\mathrm{C} 1), 52.5\left(\mathrm{OCH}_{3}\right), 123.8\left(\mathrm{C}^{\prime}{ }^{\prime}\right.$ and $\left.\mathrm{C} 8^{\prime}\right), 131.0\left(\mathrm{C}^{\prime}\right)$, 131.6 (C2'), 137.8 (C5' and C9'), 141.5 (C4'), 147.4 (C7'), $167.7(\mathrm{C}=\mathrm{O}), 200.0(\mathrm{C}=\mathrm{N})$; HRMS (ESI) $\mathrm{m} / \mathrm{z}$, calcd. for $\mathrm{C}_{14} \mathrm{H}_{15} \mathrm{~N}_{2} \mathrm{O}_{6} \mathrm{~S}_{3}^{-}: 431.0411$, found: 431.0388 .

Tetraphenylphosphonium (Z)-2-(methoxycarbonyl)3-(4-nitrophenyl)allyl-( $N$-butylsulfonyl)dithiocarbimate (2d)

Yield 75\%; oil at room temperature; IR (selected bands, ATR $) v / \mathrm{cm}^{-1} 1711(v \mathrm{C}=\mathrm{O}), 1516\left(v_{\mathrm{as}} \mathrm{NO}_{2}\right), 1381(v \mathrm{C}=\mathrm{N})$, $1342\left(v_{\text {sym }} \mathrm{NO}_{2}\right), 1259\left(v_{\text {as }} \mathrm{SO}_{2}\right), 1147\left(v_{\text {sym }} \mathrm{SO}_{2}\right), 935\left(v_{\text {as }} \mathrm{CS}_{2}\right)$; ${ }^{1} \mathrm{H}$ NMR (allyldithiocarbimate signals, $300 \mathrm{MHz}, \mathrm{CDCl}_{3}$ ) $\delta$ 0.78-0.93 (m, 3H, H8), 1.14-1.47 (m, 10H, H3-H7), 1.70-1.85 (m, 2H, H2), 3.52 (t, 2H, J 8.1 Hz, H1), 3.79 (s, $\left.3 \mathrm{H}, \mathrm{OCH}_{3}\right), 4.20$ (s, 2H, H1'), 7.56-7.70 (m*, 1H, H3'), 7.71-7.83 (m*, 2H, H5', H9'), 8.17 (d, 2H, J 8.7 Hz, H6', $\mathrm{H} 8$ '); ${ }^{13} \mathrm{C}$ NMR (allyldithiocarbimate signals, $75 \mathrm{MHz}$, $\mathrm{CDCl}_{3}$ ) $\delta 14.1$ (C8), 22.6 (C7), 23.7 (C6), 28.8 (C5), 29.1 (C4), 29.3 (C3), 31.8 (C2), 33.0 (C1'), 51.4 (C1), $52.5\left(\mathrm{OCH}_{3}\right), 123.8\left(\mathrm{C} 6^{\prime}\right.$ and $\left.\mathrm{C}^{\prime}\right), 131.0\left(\mathrm{C}^{\prime}\right), 131.5$ (C2'), 137.8 (C5' and C9'), 141.4 (C4'), 147.3 (C7'), $167.6(\mathrm{C}=\mathrm{O}), 200.0(\mathrm{C}=\mathrm{N})$; HRMS (ESI) $\mathrm{m} / \mathrm{z}$, calcd. for $\mathrm{C}_{20} \mathrm{H}_{27} \mathrm{~N}_{2} \mathrm{O}_{6} \mathrm{~S}_{3}^{-}: 487.1037$, found: 487.0995 .

Tetraphenylphosphonium (Z)-2-(methoxycarbonyl)3-[4-(trifluoromethyl)phenyl]allyl-( $N$-methylsulfonyl) dithiocarbimate (3a)

Yield 89\%; mp 136.8-138.5 ${ }^{\circ} \mathrm{C}$; IR (selected bands, ATR $v / \mathrm{cm}^{-1} 1722(v \mathrm{C}=\mathrm{O}), 1385(v \mathrm{C}=\mathrm{N}), 1261\left(v_{\mathrm{as}} \mathrm{SO}_{2}\right)$, $1151\left(v_{\text {sym }} \mathrm{SO}_{2}\right), 1063\left(v \mathrm{CF}_{3}\right), 930\left(v_{\text {as }} \mathrm{CS}_{2}\right) ;{ }^{1} \mathrm{H}$ NMR (allyldithiocarbimate signals, $300 \mathrm{MHz}, \mathrm{CDCl}_{3}$ ) $\delta 3.21$ (s, 3H, H1), 3.75 (s, 3H, $\left.\mathrm{OCH}_{3}\right), 4.17$ (s, 2H, H1'), 7.52-7.69 (m*, 5H, H3', H5', H6', H8', H9'); ${ }^{13} \mathrm{C}^{*} \mathrm{NMR}$ (allyldithiocarbimate signals, $75 \mathrm{MHz}, \mathrm{CDCl}_{3}$ ) $\delta 33.2$ (C1'), $39.1(\mathrm{C} 1), 52.4\left(\mathrm{OCH}_{3}\right), 125.5$ (q, J 3.7 Hz, C6' and C8'), 127.7 (q, J $270 \mathrm{~Hz}, \mathrm{CF}_{3}$ ), 129.9 (C2'), 130.3 (C5' and 
C9'), 130.6 (q, J 32 Hz, C7'), 138.4 (C3'), 139.3 (C4'), $167.9(\mathrm{C}=\mathrm{O}), 201.3(\mathrm{C}=\mathrm{N})$; HRMS (ESI) $\mathrm{m} / \mathrm{z}$, calcd. for $\mathrm{C}_{14} \mathrm{H}_{13} \mathrm{~F}_{3} \mathrm{NO}_{4} \mathrm{~S}_{3}^{--}: 411.9964$, found: 411.9961.

Tetraphenylphosphonium (Z)-2-(methoxycarbonyl)3-[4-(trifluoromethyl)phenyl]allyl-( $N$-ethylsulfonyl) dithiocarbimate (3b)

Yield 85\%; mp 133.8-135.2 ${ }^{\circ} \mathrm{C}$; IR (selected bands, ATR $v / \mathrm{cm}^{-1} 1718(v \mathrm{C}=\mathrm{O}), 1379(v \mathrm{C}=\mathrm{N}), 1261\left(v_{\mathrm{as}} \mathrm{SO}_{2}\right)$, $1153\left(v_{\text {sym }} \mathrm{SO}_{2}\right), 1061\left(v \mathrm{CF}_{3}\right), 930\left(v_{\text {as }} \mathrm{CS}_{2}\right) ;{ }^{1} \mathrm{H}$ NMR (allyldithiocarbimate signals, $300 \mathrm{MHz}, \mathrm{CDCl}_{3}$ ) $\delta 1.29$ (t, 3H, J 7.5 Hz, H2), 3.54 (q, 2H, J 7.5 Hz, H1), 3.76 (s, $3 \mathrm{H}, \mathrm{OCH}_{3}$ ), 4.19 (s, 2H, H1'), 7.54-7.70 (m*, 5H, H3', H5', H6', H8', H9'); ${ }^{13} \mathrm{C}$ NMR (allyldithiocarbimate signals, $\left.75 \mathrm{MHz}, \mathrm{CDCl}_{3}\right) \delta 8.4(\mathrm{C} 2), 33.1\left(\mathrm{Cl}^{\prime}\right.$ ) $), 45.4(\mathrm{C} 1)$, $52.3\left(\mathrm{OCH}_{3}\right), 125.5$ (q, J $3.8 \mathrm{~Hz}, \mathrm{C}^{\prime}$ ' and $\left.\mathrm{C} 8^{\prime}\right), 127.6$ (q, $\left.J 270 \mathrm{~Hz}, \mathrm{CF}_{3}\right), 129.9$ (C2'), 130.3 (C5' and C9'), 130.5 (q, J31.5 Hz, C7'), 138.4 (C3'), 139.0 (C4'), 167.9 (C=O), $200.3\left(\mathrm{C}=\mathrm{N}\right.$ ); HRMS (ESI) $\mathrm{m} / z$, calcd. for $\mathrm{C}_{15} \mathrm{H}_{15} \mathrm{~F}_{3} \mathrm{NO}_{4} \mathrm{~S}_{3}{ }^{-}$: 426.0121, found: 426.0142 .

Tetraphenylphosphonium (Z)-2-(methoxycarbonyl)3-[4-(trifluoromethyl)phenyl]allyl-( $N$-butylsulfonyl) dithiocarbimate (3c)

Yield 79\%; mp 119.2-120.8 ${ }^{\circ} \mathrm{C}$; IR (selected bands, ATR $v / \mathrm{cm}^{-1} 1705(v \mathrm{C}=\mathrm{O}), 1396(v \mathrm{C}=\mathrm{N}), 1255\left(v_{\mathrm{as}} \mathrm{SO}_{2}\right)$, $1157\left(v_{\mathrm{sym}} \mathrm{SO}_{2}\right), 1065\left(v \mathrm{CF}_{3}\right), 926\left(v_{\mathrm{as}} \mathrm{CS}_{2}\right) ;{ }^{1} \mathrm{H} \mathrm{NMR}$ (allyldithiocarbimate signals, $\left.300 \mathrm{MHz}, \mathrm{CDCl}_{3}\right) \delta 0.86$ (t, $3 \mathrm{H}, J 7.2 \mathrm{~Hz}, \mathrm{H} 4$ ), 1.38 (sext, 2H, J 7.5 Hz, H3), 1.73-1.84 (m, 2H, H2), 3.54 (t, $2 \mathrm{H}, J 8.1 \mathrm{~Hz}, \mathrm{H} 1), 3.76\left(\mathrm{~s}, 3 \mathrm{H}, \mathrm{OCH}_{3}\right)$, 4.19 (s, 2H, H1'), 7.52-7.70 (m*, 5H, H3', H5', H6', H8', $\mathrm{H} 9$ '); ${ }^{13} \mathrm{C}$ NMR (allyldithiocarbimate signals, $75 \mathrm{MHz}$, $\mathrm{CDCl}_{3}$ ) $\delta 13.9$ (C4), $22.0(\mathrm{C} 3), 25.8$ (C2), 33.1 (C1'), $51.0(\mathrm{C} 1), 52.4\left(\mathrm{OCH}_{3}\right), 125.5$ (q, J $3.8 \mathrm{~Hz}, \mathrm{C}^{\prime}$ ' and $\mathrm{C} 8$ '), 127.7 (q, $\left.J 270.0 \mathrm{~Hz}, \mathrm{CF}_{3}\right), 129.9$ (C2'), 130.0 (C5' and C9'), 130.5 (q, J 31.5 Hz, C7'), 138.4 (C3'), 139.1 (C4'), $167.9(\mathrm{C}=\mathrm{O}), 200.3(\mathrm{C}=\mathrm{N})$; HRMS (ESI) $\mathrm{m} / \mathrm{z}$, calcd. for $\mathrm{C}_{17} \mathrm{H}_{19} \mathrm{~F}_{3} \mathrm{NO}_{4} \mathrm{~S}_{3}^{-}: 454.0434$, found: 454.0408 .

Tetraphenylphosphonium (Z)-2-(methoxycarbonyl)3-[4-(trifluoromethyl)phenyl]allyl-( $N$-octylsulfonyl) dithiocarbimate (3d)

Yield $72 \%$; oil at room temperature; IR (selected bands, ATR $v / \mathrm{cm}^{-1} 1711(v \mathrm{C}=\mathrm{O}), 1381(v \mathrm{C}=\mathrm{N}), 1259\left(v_{\mathrm{as}} \mathrm{SO}_{2}\right)$, $1163\left(v_{\text {sym }} \mathrm{SO}_{2}\right), 1065\left(v \mathrm{CF}_{3}\right), 933\left(v_{\mathrm{as}} \mathrm{CS}_{2}\right) ;{ }^{1} \mathrm{H}$ NMR (allyldithiocarbimate signals, $300 \mathrm{MHz}, \mathrm{CDCl}_{3}$ ) $\delta 0.79-0.93$ (m, 3H, H8), 1.12-1.45 (m, 10H, H3-H7), 1.72-1.88 (m, 2H, $\mathrm{H} 2), 3.53$ (t, $2 \mathrm{H}, J 7.8 \mathrm{~Hz}, \mathrm{H} 1), 3.76$ (s, $\left.3 \mathrm{H}, \mathrm{OCH}_{3}\right), 4.19$ (s, 2H, H1'), 7.56-7.70 (m, 5H, H3', H5', H6', H8', H9'); ${ }^{13} \mathrm{C}$ NMR (allyldithiocarbimate signals, $75 \mathrm{MHz}, \mathrm{CDCl}_{3}$ ) $\delta 14.1$ (C8), 22.6 (C7), 23.7 (C6), 28.8 (C5), 29.1 (C4),
$29.3(\mathrm{C} 3), 31.8(\mathrm{C} 2), 33.0\left(\mathrm{C} 1\right.$ ') $, 51.3(\mathrm{C} 1), 52.3\left(\mathrm{OCH}_{3}\right)$, $125.4\left(\mathrm{q}, J 3.7 \mathrm{~Hz}, \mathrm{C}^{\prime}\right.$ ' and C8'), 127.6 (q, $J 271.0 \mathrm{~Hz}$, $\mathrm{CF}_{3}$ ), $129.4\left(\mathrm{C}^{\prime}{ }^{\prime}\right), 130.3$ (C5' and C9') 130.5 (q, J31.5 Hz, C7'), 138.3 (C3'), 139.0 (C4'), 167.8 (C=O), $200.2(\mathrm{C}=\mathrm{N})$; HRMS (ESI) $\mathrm{m} / z$, calcd. for $\mathrm{C}_{21} \mathrm{H}_{27} \mathrm{~F}_{3} \mathrm{NO}_{4} \mathrm{~S}_{3}{ }^{-}: 510.1060$, found: 510.0993 .

\section{X-ray crystallography}

Yellow crystals of the compound 2a were obtained by slow evaporation of a dichloromethane/ethanol solution (1:1) and a few drops of water at $25^{\circ} \mathrm{C}$. The diffraction measurement was achieved at room temperature with a Bruker APEX II CCD diffractometer using Mo K $\alpha$ radiation $(\lambda 0.71073 \AA$ ). Data collection, cell refinement and data reduction were made using APEX2 software. ${ }^{18}$ Final unit cell parameters based on all reflections were obtained by least squares refinement. The data were integrated via SAINT. ${ }^{19}$ Lorentz and polarization effect and multi-scan absorption corrections were applied with SADABS. ${ }^{20}$ The structure was solved with SHELXS97 using direct methods and refined by full-matrix least-square methods against $\mathrm{F}^{2}$ (SHELXL-2014). ${ }^{21,22}$ All hydrogen atoms were stereochemically positioned and refined with the riding model. The crystal structure was refined as a non-merohedral twin with a 2 -fold axis rotation around the $c^{*}$ axis ( $c$-axis of the reciprocal space) and a BASF parameter of 0.04767. Structural representations were drawn using ORTEP- $3^{23}$ and MERCURY. ${ }^{24}$ The program WinGX was used to prepare materials for publication. ${ }^{23}$ The data collection and experimental details for compound $\mathbf{2 a}$ can be found in the Table 1 .

\section{Biological assay}

The culture medium, prepared with agar-agar (Vetec, Duque de Caxias, Brazil) and distilled water, was sterilized by autoclaving at $121{ }^{\circ} \mathrm{C}$ for $25 \mathrm{~min}$. Each compound was dissolved in dimethyl sulfoxide and Tween $80(0.75 \mathrm{~mL}$ each) and the solutions were homogeneously mixed with the agar-agar suspension $(100 \mathrm{~mL})$ and verted into sterile Petri dishes. The final concentrations for each tested substance in the culture medium were equal to $15,30,60$, 120, 240, 360, 480 and $960 \mu \mathrm{mol} \mathrm{L}{ }^{-1}$. Each treatment had three replicates. The zinc bis-dimethyldithiocarbamate (97\%, Sigma-Aldrich, St. Louis, USA) was included as a positive control in the tests at $120 \mu \mathrm{mol} \mathrm{L}^{-1}$ and the results were compared by the Tukey's test $(p \leq 0.05)$.

Phakopsora pachyrhizi and Hemileia vastatrix spores were isolated from infected soybean (Glycine max, cultivar: Monarca) and coffee (Coffea arabica, cultivar: Red 
Table 1. Crystallographic data and details of diffraction experiments for compound 2a

\begin{tabular}{|c|c|}
\hline Empirical formula & $\mathrm{C}_{37} \mathrm{H}_{33} \mathrm{NO}_{6} \mathrm{PS}_{3}$ \\
\hline Formula weight $/\left(\mathrm{g} \mathrm{mol}^{-1}\right)$ & 728.80 \\
\hline Temperature / K & $296(2)$ \\
\hline Crystal system & monoclinic \\
\hline Space group & $\mathrm{P} 2_{1} / \mathrm{c}$ \\
\hline \multicolumn{2}{|l|}{ Unit cell dimensions } \\
\hline$a / \AA$ & $7.808(1)$ \\
\hline$b / \AA$ & $14.182(1)$ \\
\hline$c / \AA$ & $32.506(2)$ \\
\hline$\beta /$ degree & $93.209(2)$ \\
\hline Volume / $\AA^{3}$ & $3594.2(3)$ \\
\hline $\mathrm{Z}$ & 4 \\
\hline Calculated density / $\left(\mathrm{g} \mathrm{cm}^{-3}\right)$ & 1.347 \\
\hline$\mu / \mathrm{mm}^{-1}$ & 0.299 \\
\hline $\mathrm{T}_{\min } / \mathrm{T}_{\max }$ & $0.902 / 0.926$ \\
\hline $\mathrm{F}(000)$ & 1520 \\
\hline Crystal size / mm & $0.386 \times 0.288 \times 0.256$ \\
\hline$\theta$ range / degree & 0.627 to 26.403 \\
\hline Limiting indices & $\begin{array}{c}-5 \leq h \leq 9 ;-17 \leq k \leq 13 \\
-40 \leq l \leq 38\end{array}$ \\
\hline Reflections collected & 19122 \\
\hline Independent reflections & $7358[\mathrm{R}(\mathrm{int})=0.0365]$ \\
\hline Goodness-of-fit & 1.087 \\
\hline Data / restraints / parameters & 5872 / 0 / 444 \\
\hline$R$ indices $[\mathrm{I}>2 \sigma(\mathrm{I})]$ & $\mathrm{R}=0.0853, w \mathrm{R}=0.2806$ \\
\hline $\mathrm{R}$ indices (all data) & $\mathrm{R}=0.1030, w \mathrm{R}=0.3057$ \\
\hline Largest diff. peak and hole / $\left(\mathrm{e} \AA^{-3}\right)$ & 1.097 and -0.701 \\
\hline
\end{tabular}

$\mathrm{R}=\sum\left(\left|\mathrm{F}_{0}\right|-\left|\mathrm{F}_{\mathrm{c}}\right|\right) / \sum\left|\mathrm{F}_{0}\right| ; \mathrm{wR}=\left[\sum \mathrm{w}\left(\left|\mathrm{F}_{0}^{2}\right|-\left|\mathrm{F}_{\mathrm{c}}^{2}\right|\right) / \sum \mathrm{w}\left|\mathrm{F}_{0}^{2}\right|^{2}\right]^{1 / 2}$.

$\mu$ : absorption coefficient; $\mathrm{F}(000)$ : structure factor in the zeroth-order case; $\mathrm{R}$ (int): internal R-value.

Caturra) leaves, respectively. The spore concentrations were adjusted to $1 \times 10^{5}$ spore $\mathrm{mL}^{-1}$ in distilled water, with the aid of a hemocytometer. Then, $100 \mu \mathrm{L}$ of these suspensions were added to each Petri dish and homogeneously spread over the culture medium. After $24 \mathrm{~h}$ of incubation at $25^{\circ} \mathrm{C}$, each plate was divided into four quadrants and 25 spores per quadrant were examined under the microscope to assess germination. The averages were compared to the negative control (prepared using dimethyl sulfoxide, Tween 80 and agar-agar only) and the inhibition percentages were calculated. The results were statistically analyzed by nonlinear regression using the concentration logarithm versus percent inhibition results. The activities were submitted to analyses of variance. The needed concentrations to achieve 50 and $90 \%$ of inhibition of spore germination $\left(\mathrm{IC}_{50}\right.$ and $\mathrm{IC}_{90}$ values) were calculated for each compound from the regression equations. ${ }^{25,26}$ The whole experiment was repeated, and the results confirmed the first data set (see Tables S1, S2 and S3 in the Supplementary Information).

\section{Results and Discussion}

\section{Syntheses and characterization}

Twelve novel allyldithiocarbimate anions were prepared by the reactions between aliphatic sulfonyldithiocarbimates and allylic bromides derived from MBH adducts (Scheme 3). There is only one paper ${ }^{8}$ in the literature reporting the synthesis of allyldithiocarbimates, though these were derived from aromatic sulfonylditiocarbimates. Using a similar approach, here we demonstrate that the aliphatic sulfonyldithiocarbimates also act as nucleophiles, reacting in a fast way with the allylic bromides at room temperature (less than $15 \mathrm{~min}$ ) furnishing the allyldithiocarbimates in good yields (72-82\%).

As the resulting potassium salts are water soluble and not easily purifiable, the new compounds were isolated as tetraphenylphosphonium salts. The new salts are yellow solids (1a-c, 2a-c and 3a-c) or yellowish viscous oils (1d, 2d and $\mathbf{3 d}$ ) at room temperature, soluble in dimethyl sulfoxide, dimethylformamide, acetone, ethyl acetate, ethanol, chloroform and dichloromethane, with very low solubility in diethyl ether, hexanes and water. Their molecular formulae were confirmed by HRMS, which presented the expected peak for the tetraphenylphosphonium ion in the positive mode, and the molecular ion peaks of each allyldithiocarbimate in the negative mode. The ${ }^{1} \mathrm{H}$ NMR spectra integration curves were in accordance with a 1:1 proportion between each anion and the tetraphenylphosphonium cation.

Characteristic bands in the IR spectra indicated the presence of the most relevant groups within each structure. All spectra presented the $\alpha, \beta$-unsaturated ester carbonyl band at around $1710 \mathrm{~cm}^{-1}$, the $\mathrm{C}=\mathrm{N}$ and $\mathrm{CS}_{2}$ stretching bands at ca. 1380 and $930 \mathrm{~cm}^{-1}$, respectively, and the strong bands due to the $\mathrm{SO}_{2}$ group at ca. 1260 and $1150 \mathrm{~cm}^{-1}$. The $\mathrm{C}=\mathrm{N}$ bands were shifted to higher wavenumber values with respect to the parent potassium dithiocarbimates data ( $v C N$ 1260-1300 $\left.\mathrm{cm}^{-1}\right){ }^{12-14}$ This shift can be explained by the partial loss of conjugation within the $\mathrm{NCS}_{2}$ system. Consequently, the $\mathrm{CS}_{2}$ bands had the opposite shift (potassium dithiocarbimates $\mathrm{vCS}_{2} 945-979 \mathrm{~cm}^{-1}$ ). Compounds 2a-d and 3a-d IR spectra presented additional bands due to the nitro (ca. 1515 and $1340 \mathrm{~cm}^{-1}$ ) and trifluoromethyl (ca. $1065 \mathrm{~cm}^{-1}$ ) groups. 
The signals due to the alkyl groups in the ${ }^{13} \mathrm{C}$ NMR spectra had similar chemical shift values when compared to the parent potassium dithiocarbimates. ${ }^{12-14}$ One important change was observed on the signal of the carbon atom of the $\mathrm{NCS}_{2}$ group, which was shifted from ca. $\delta 224$ in the spectra of the precursors to ca. $\delta 200$ in the spectra of the allyldithiocarbimates. This shift is in agreement with the IR data, and is due to the greater double bond character of the $\mathrm{CN}$ bond in the allyldithiocarbimates. Two signals in higher field (ca. $\delta 33$ and 52), present in all spectra, were due to the $\mathrm{CH}_{2}$ and $\mathrm{OCH}_{3}$ carbon atoms of the $\mathrm{MBH}$ moiety, and the ester carbonyl signal was observed at ca. $\delta 168$. The spectra of compounds $\mathbf{3 a}$-d showed a quartet centered at ca. $\delta 128(\mathrm{~J} 270 \mathrm{~Hz})$ due to the $\mathrm{CF}_{3}$ group, which also caused the split in the aromatic signals at around $\delta 130.5$ $\left({ }^{2} J 31.5 \mathrm{~Hz}, \mathrm{C}^{\prime}\right.$ ') and $\delta 125.5$ (' $J 3.8 \mathrm{~Hz}, \mathrm{C} 6$ ' and $\mathrm{C} 8$ ').

In order to investigate the stereochemistry of the allyldithiocarbimates, NOESY experiments were performed. A correlation between the hydrogens $\mathrm{H} 1$ ' and H9' signals indicated a spatial proximity coherent with a $Z$ configuration with respect to the $\mathrm{C} 2$ '- $\mathrm{C} 3$ ' double bond.

\section{X-ray crystallography}

Compound 2a crystallizes in the monoclinic space group $\mathrm{P}_{1} / \mathrm{c}$. The asymmetric unit contains one anion and one cation (Figure 2). Single crystal X-ray diffraction data confirmed the $(Z)$-stereochemistry for the allyldithiocarbimate anion.

Selected bond lengths and torsion angles are summarized in Table 2. The bond length C2'-C3' $[1.329(8) \AA]$ is consistent with a normal $\mathrm{C}=\mathrm{C}$ double bond $(1.34 \AA$ ). The
Table 2. Selected bond lengths and torsion angles for compound 2a

\begin{tabular}{|c|c|}
\hline \multicolumn{2}{|c|}{ Bond length / $̊$} \\
\hline $\mathrm{S} 1-\mathrm{C} 1$ & $1.735(7)$ \\
\hline S1-N1 & $1.623(5)$ \\
\hline $\mathrm{C} 2-\mathrm{N} 1$ & $1.306(7)$ \\
\hline $\mathrm{C} 2-\mathrm{S} 3$ & $1.689(5)$ \\
\hline $\mathrm{C} 2-\mathrm{S} 2$ & $1.777(5)$ \\
\hline $\mathrm{S} 2-\mathrm{C} 1$ ' & $1.830(6)$ \\
\hline $\mathrm{C} 1^{\prime}-\mathrm{C} 2{ }^{\prime}$ & $1.515(7)$ \\
\hline $\mathrm{C} 2{ }^{\prime}-\mathrm{C} 3{ }^{\prime}$ & $1.329(8)$ \\
\hline C3'-C4' & $1.464(9)$ \\
\hline $\mathrm{C} 2{ }^{\prime}-\mathrm{C} 3$ & $1.506(8)$ \\
\hline $\mathrm{C} 3-\mathrm{O} 4$ & $1.341(8)$ \\
\hline $\mathrm{C} 3-\mathrm{O} 3$ & $1.191(8)$ \\
\hline $\mathrm{O} 4-\mathrm{C} 4$ & $1.453(9)$ \\
\hline C7'-N1 & $1.469(9)$ \\
\hline \multicolumn{2}{|c|}{ Torsion angle / degree } \\
\hline $\mathrm{C} 2-\mathrm{S} 2-\mathrm{C} 1^{\prime}-\mathrm{C} 2^{\prime}$ & 111.66 \\
\hline $\mathrm{S} 2-\mathrm{C} 1^{\prime}-\mathrm{C} 2^{\prime}-\mathrm{C} 3^{\prime}$ & -121.15 \\
\hline $\mathrm{S} 2-\mathrm{C} 1{ }^{\prime}-\mathrm{C} 2{ }^{\prime}-\mathrm{C} 3$ & 60.92 \\
\hline
\end{tabular}

C2'-C3 bond $[1.506(8) \AA]$ is in the range of a $\mathrm{Csp}^{2}-\mathrm{Csp}^{3}$ sigma bond $(1.50 \AA)$. The bond lengths (Table 2) within the methoxycarbonyl group (C3-O3, C3-O4 and C4-O4) are within the range reported for the analogues derived from aromatic sulfonildithiocarbimates. ${ }^{8}$ The N1-C2 bond distance $[1.306(7) \AA]$ is slightly longer than the value of $1.279 \AA$ for a typical $\mathrm{N}(\mathrm{sp} 2)=\mathrm{C}(\mathrm{sp} 2)$ double

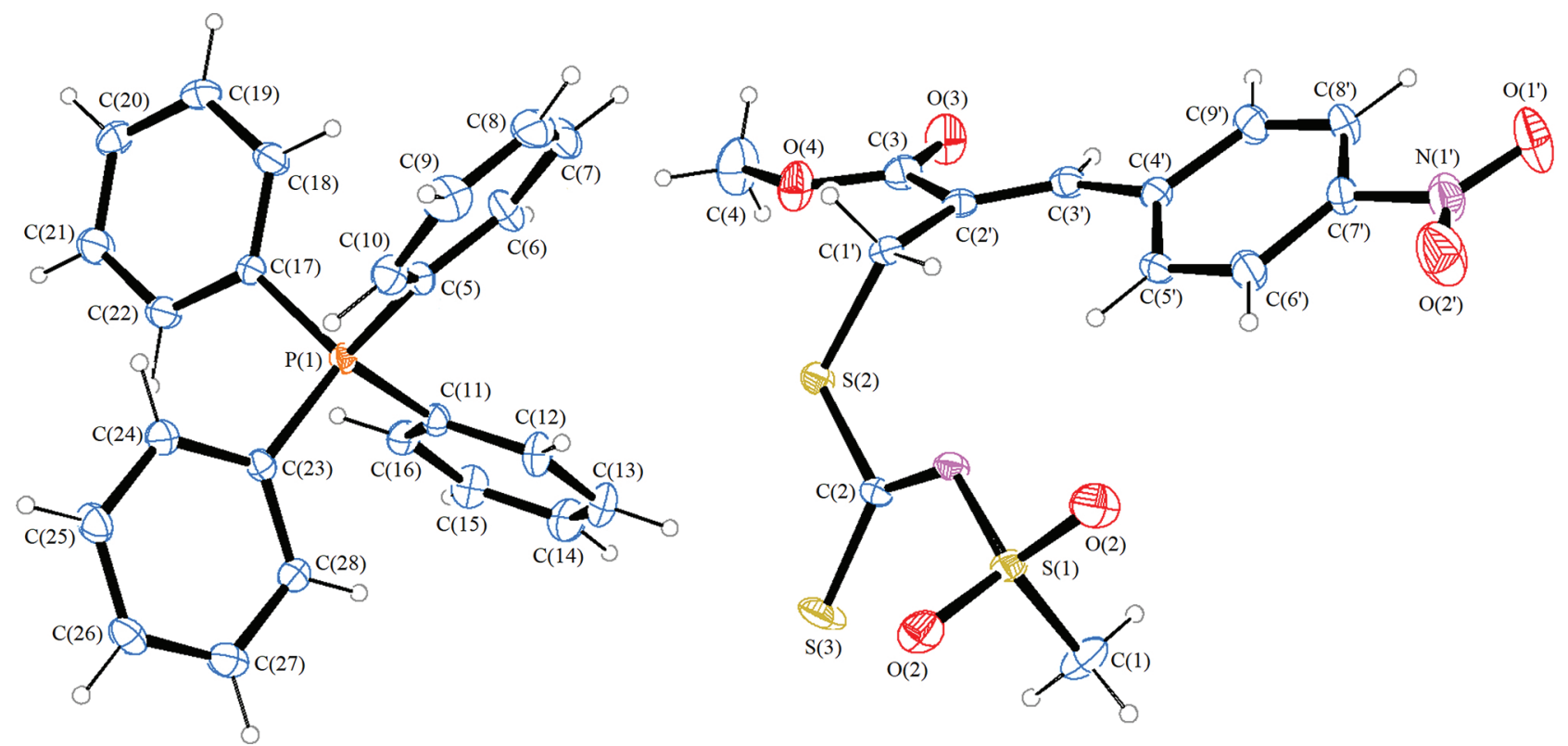

Figure 2. ORTEP view of compound 2a with atom-numbering scheme and displacement ellipsoids drawn with $20 \%$ probability level, and numbering for the X-ray data analyses. 
bond. ${ }^{27}$ In addition, the $\mathrm{C} 2-\mathrm{S} 2$ bond length $[1.777(5) \AA]$ is significantly longer than the $\mathrm{C} 2-\mathrm{S} 3$ distance $[1.689(5) \AA]$. The $\mathrm{C} 2-\mathrm{S} 2$ distance is shorter than a $\mathrm{C}-\mathrm{S}$ single bond and the $\mathrm{C} 2-\mathrm{S} 3$ is slightly longer than a typical $\mathrm{C}=\mathrm{S}$ double bond. ${ }^{27}$ These facts are due to the conjugated $\mathrm{NCS}_{2}$ system and agree with the IR and NMR data.

Three planar fragments characterize the anion: $\mathrm{S} 1 / \mathrm{N} 1 / \mathrm{C} 2 / \mathrm{S} 2 / \mathrm{S} 3 / \mathrm{C} 1$ ', $\mathrm{C} 2{ }^{\prime} / \mathrm{C} 3 / \mathrm{O} 3 / \mathrm{O} 4 / \mathrm{C} 4 / \mathrm{C} 1$ ' and the phenyl ring. The angle between the first two fragments is $63.7(2)^{\circ}$ and between the second and third fragments is $25.5(3)^{\circ}$. The torsion angles around the bonds $\mathrm{C} 1{ }^{\prime}-\mathrm{C} 2$ ' and $\mathrm{S} 2-\mathrm{C} 1$ ' describe the orientation of these fragments (Table 2).

The phosphorus atom in the tetraphenylphosphoniun cation present tetrahedral distorted geometry, with $\mathrm{P}-\mathrm{C}$ and $\mathrm{C}-\mathrm{C}$ bond lengths in the range from 1.787(6) to 1.797(5) $\AA$ and 1.360(10) to 1.408(8) $\AA$, respectively. The $\mathrm{C}-\mathrm{P}-\mathrm{C}$ angles in the four phenyl rings go from 107.4(2) to $111.5(3)^{\circ}$. These values are comparable to those found in other structures with the same cation. ${ }^{8,28}$

The crystal packing is mainly stabilized by the electrostatic interactions between oppositely charged ions (Figure 3). In addition, there are four intramolecular contacts in the anion, and intermolecular interactions between cations and anions $(\mathrm{C}-\mathrm{H} \cdots \mathrm{O})$, between anions $(\mathrm{C}-\mathrm{H} \cdots \mathrm{S})$ and between cations $(\mathrm{C}-\mathrm{H} \cdots \mathrm{Cg})$ (Table 3). The $\mathrm{C} 16-\mathrm{H} 16 \cdots \mathrm{O} 1, \mathrm{C} 20-\mathrm{H} 20 \cdots \mathrm{O} 2$ and $\mathrm{C} 1{ }^{\prime}-\mathrm{H} 1{ }^{\prime} 1 \cdots \mathrm{S} 3$ intermolecular interactions form ribbons in the $a$-axis direction (Figure 3). The $\mathrm{C} 25-\mathrm{H} 25 \cdots \mathrm{Cg}$ interactions connect these ribbons forming chains along $b$-axis.

\section{Antifungal activity}

The influences of the twelve allyldithiocarbimate salts shown in Scheme 3 were investigated, in vitro, on the

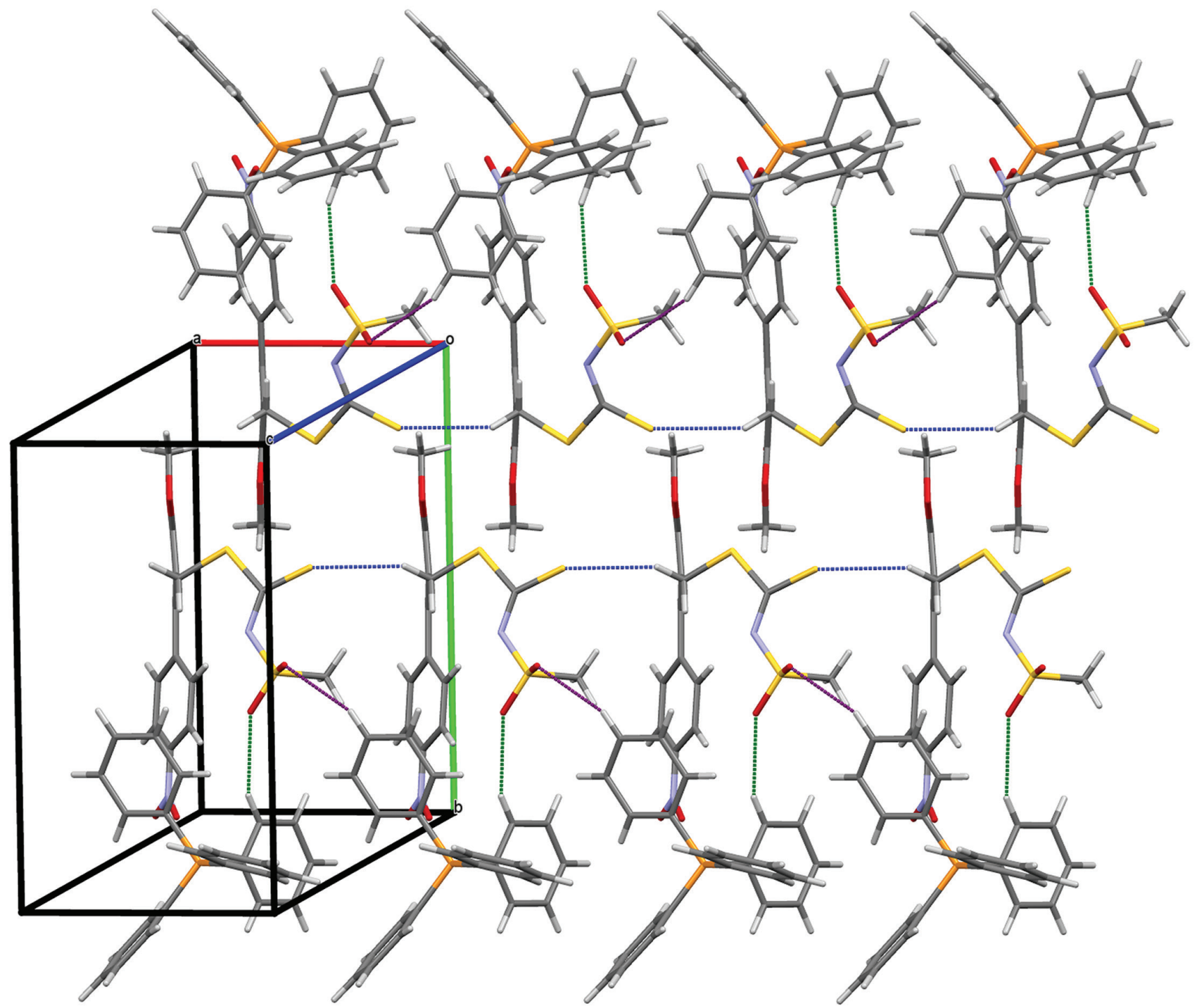

Figure 3. Structural packing of compound 2a in the $a$-axis direction. Dashed lines indicate $\mathrm{C} 16-\mathrm{H} 16 \cdots \mathrm{O} 1, \mathrm{C} 20-\mathrm{H} 20 \cdots \mathrm{O} 2$ and $\mathrm{C} 1^{\prime}-\mathrm{H} 1{ }^{\prime} 1 \cdots \mathrm{S} 3$ hydrogen bonds . 
Table 3. Geometric parameters for intramolecular and intermolecular interactions in compound $2 \mathbf{a}$ crystal

\begin{tabular}{|c|c|c|c|c|}
\hline Donor-H $\cdots$ acceptor & $\mathrm{d}(\mathrm{D}-\mathrm{H}) / \AA$ & $\mathrm{d}(\mathrm{H} \cdots \mathrm{A}) / \AA$ & $\mathrm{d}(\mathrm{D} \cdots \mathrm{A}) / \AA$ & $<$ (DHA) / degree \\
\hline $\mathrm{C} 1-\mathrm{H} 1 \mathrm{~A} \cdots \mathrm{S} 3$ & 0.96 & 2.78 & $3.419(9)$ & 125 \\
\hline $\mathrm{C} 1{ }^{\prime}-\mathrm{H} 1{ }^{\prime} 2 \cdots \mathrm{N} 1$ & 0.97 & 2.40 & $2.940(7)$ & 115 \\
\hline $\mathrm{C} 3^{\prime}-\mathrm{H} 3^{\prime} \cdots \mathrm{O} 3$ & 0.93 & 2.33 & $2.759(8)$ & 108 \\
\hline $\mathrm{C}^{\prime}-\mathrm{H} 5^{\prime} \cdots \mathrm{N} 1$ & 0.93 & 2.42 & $3.345(8)$ & 173 \\
\hline $\mathrm{C} 16-\mathrm{H} 16 \cdots \mathrm{O} 1^{\mathrm{i}}$ & 0.93 & 2.56 & $3.423(7)$ & 154 \\
\hline $\mathrm{C} 20-\mathrm{H} 20 \cdots \mathrm{O} 2^{\mathrm{ii}}$ & 0.93 & 2.56 & $3.421(9)$ & 155 \\
\hline $\mathrm{C} 1^{\prime}-\mathrm{H} 1^{\prime} 1 \cdots \mathrm{S} 3^{\mathrm{iii}}$ & 0.97 & 2.86 & $3.675(6)$ & 143 \\
\hline $\mathrm{C} 25-\mathrm{H} 25 \cdots \mathrm{Cg}^{\mathrm{iv}}$ & 0.93 & 2.89 & $3.685(8)$ & 144 \\
\hline
\end{tabular}

Symmetry codes: (i) x, $1+\mathrm{y}, \mathrm{z}$; (ii) $-1+\mathrm{x}, 1+\mathrm{y}, \mathrm{z}$; (iii) $-1+\mathrm{x}, \mathrm{y}, \mathrm{z}$; (iv) $1-\mathrm{x}, 1 / 2+\mathrm{y}, 1 / 2-\mathrm{z}$. Cg is the C23-C28 ring.

germination of $H$. vastatrix and $P$. pachyrhizi spores. The new compounds showed comparable activities to those presented by the ZDMC, the pure active principle of the protectant fungicide Ziram at $120 \mu \mathrm{mol} \mathrm{L}^{-1}$ (Figures 4 and 5). ZDMC and the new allyldithiocarbimate salts were more active against $P$. pachyrhizi than against $H$. vastatrix. The results also indicated that the structural differences on the allyldithiocarbimate interfere with their activities (Figures 4 and 5).

The new compounds were tested at various concentrations ranging from 15 to $960 \mu \mathrm{mol} \mathrm{L}^{-1}$, reaching $100 \%$ of inhibition of the spore germination of both fungi below the higher concentration tested. The inhibition percentages were correlated to the logarithm of the allyldithiocarbimate salts concentrations, furnishing the curve equations shown in Tables 4 and 5. All equations presented coefficient of determination values $\left(\mathrm{r}^{2}\right)$ very close to 1.0. The $F$ value and Prob $>F$ statistics were used to test

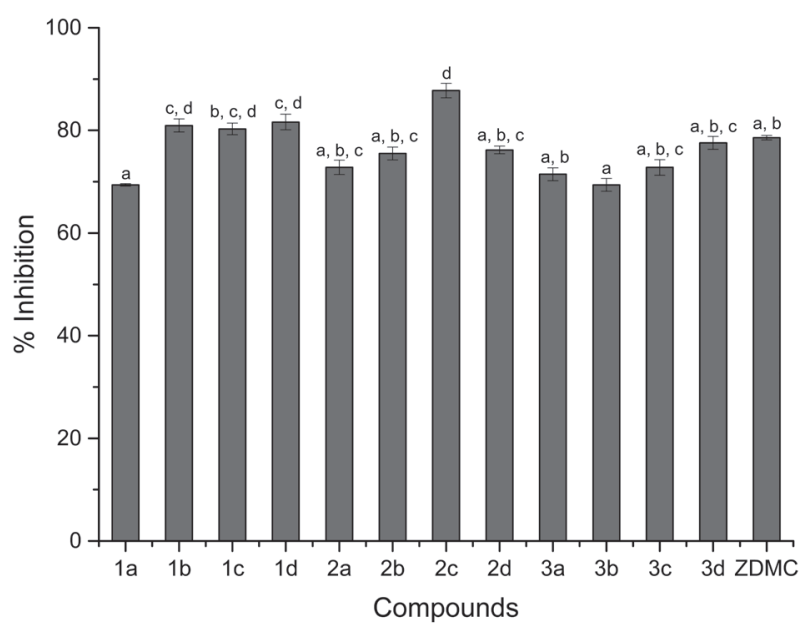

Figure 4. Inhibition (in percentage) of P. pachyrhizi spore germination caused by the new allylditiocarbimate salts compared to the positive control, zinc bis-dimethyldithiocarbamate (ZDMC) at $120 \mu \mathrm{mol} \mathrm{L}^{-1}$. Values followed by the same letter do not differ at the $5 \%$ level of significance by the Tukey's test. the overall significance of the regression model. The results showed that the choice of the sigmoidal dose-response model was adequate with a high level of significance (Prob $>F$ of less than 0.01). Figure 6 exemplifies the dose response curves obtained. In order to confirm the results, the whole experiment was repeated in different days. The data were reproductible as shown in Figure 6.

The necessary concentrations to inhibit 50 and $90 \%$ of spore germination $\left(\mathrm{IC}_{50}\right.$ and $\mathrm{IC}_{90}$ values, respectively) were calculated from the equations shown in Tables 4 and 5. The results are listed in Table 6. The complete set of results confirmed that the new compounds are significantly more active against $P$. pachyrhizi than against $H$. vastatrix, both at lower and higher concentration ranges.

It is also clear that the molecular differences within the allyldithiocarbimate anions interfere on the activity, as the various combinations of R (a-d) and R' (1-3) groups lead to different results (Table 6). With respect to the alkyl

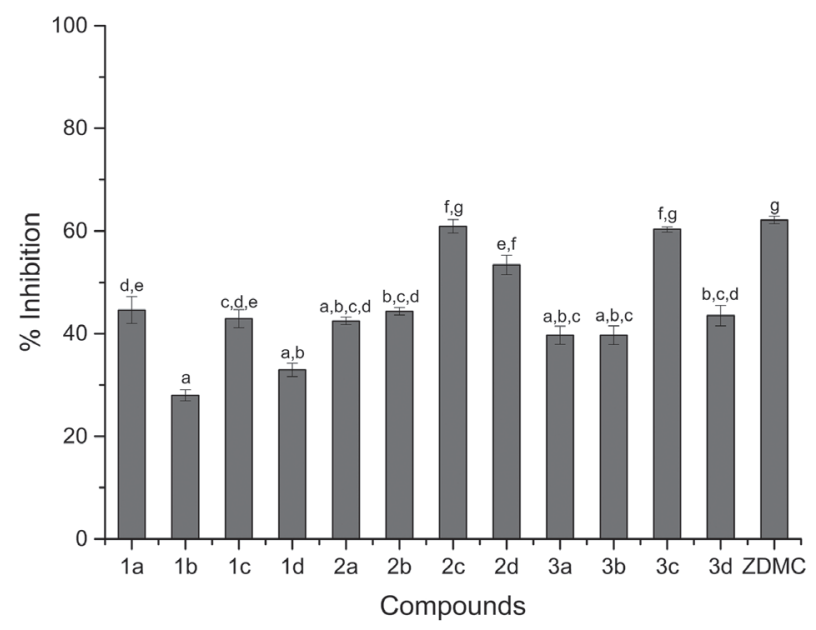

Figure 5. Inhibition (in percentage) of $H$. vastatrix spore germination caused by the new allylditiocarbimate salts compared to the positive control, zinc bis-dimethyldithiocarbamate (ZDMC) at $120 \mu \mathrm{mol} \mathrm{L}^{-1}$. Values followed by the same letter do not differ at the $5 \%$ level of significance by the Tukey's test. 
Table 4. Regression equation for the inhibition of $P$. pachyrhizi spore germination versus the log of the allyldithiocarbimate salts concentrations, equation parameters and statistical data

\begin{tabular}{|c|c|c|c|c|c|c|c|}
\hline \multirow[t]{2}{*}{ Compound } & \multicolumn{4}{|c|}{ Equation: $\mathrm{y}=\mathrm{A} 1+\frac{(\mathrm{A} 2-\mathrm{A} 1)}{\left(1+10^{(\mathrm{LOGx} 0-\mathrm{x}) \mathrm{p})}\right)}$} & \multicolumn{3}{|c|}{ Statistical data } \\
\hline & $\mathrm{A} 1$ & $\mathrm{~A} 2$ & LOGx0 & $\mathrm{p}$ & $r^{2}$ & $F$ & Prob $>F$ \\
\hline $1 \mathbf{a}$ & 13.08 & 100.74 & -1.01 & 2.25 & 0.9822 & 442.89 & $<0.0001$ \\
\hline $1 \mathrm{~b}$ & 10.91 & 99.08 & -1.13 & 2.42 & 0.9921 & 1077.50 & $<0.0001$ \\
\hline $1 c$ & -503.64 & 112.63 & -3.43 & 0.51 & 0.9819 & 1197.90 & $<0.0001$ \\
\hline 1d & 15.66 & 102.16 & -1.38 & 1.21 & 0.9832 & 1299.22 & $<0.0001$ \\
\hline $2 \mathbf{a}$ & 22.03 & 101.43 & -1.04 & 2.19 & 0.9936 & 1676.69 & $<0.0001$ \\
\hline $2 b$ & 3.89 & 107.69 & -1.23 & 0.99 & 0.9835 & 854.70 & $<0.0001$ \\
\hline $2 \mathrm{c}$ & -3.30 & 101.53 & -1.41 & 1.51 & 0.9954 & 2779.41 & $<0.0001$ \\
\hline $2 d$ & -4.65 & 100.29 & -1.25 & 1.65 & 0.9921 & 1066.90 & $<0.0001$ \\
\hline $3 \mathbf{a}$ & 19.20 & 104.14 & -1.05 & 1.43 & 0.9909 & 1391.44 & $<0.0001$ \\
\hline $3 b$ & 15.03 & 108.62 & -1.03 & 1.12 & 0.9873 & 1027.00 & $<0.0001$ \\
\hline $3 c$ & 29.78 & 104.31 & -1.03 & 1.53 & 0.9936 & 1667.36 & $<0.0001$ \\
\hline 3d & 1.90 & 105.51 & -1.25 & 1.13 & 0.9864 & 939.17 & $<0.0001$ \\
\hline
\end{tabular}

$\mathrm{y}$ : inhibition percentage; A1: bottom asymptote; A2: top asymptote; LOGx0: center; $\mathrm{x}: \log _{10}$ [concentration]; p: hill slope; $\mathrm{r}^{2}$ : coefficient of determination; $F$ : value obtained by the $F$-test; Prob $>F=$ probability value $(p$ model, $F<0.0001)$.

Table 5. Regression equation for the inhibition of $H$. vastatrix spore germination versus the log of the allyldithiocarbimate salts concentrations, equation parameters and statistical data

\begin{tabular}{|c|c|c|c|c|c|c|c|}
\hline \multirow[t]{2}{*}{ Compound } & \multicolumn{4}{|c|}{ Equation: $\mathrm{y}=\mathrm{A} 1+\frac{(\mathrm{A} 2-\mathrm{A} 1)}{\left(1+10^{(\mathrm{LOGx} 0-\mathrm{x}) \mathrm{p})}\right)}$} & \multicolumn{3}{|c|}{ Statistical data } \\
\hline & A1 & $\mathrm{A} 2$ & LOGx0 & $\mathrm{p}$ & $r^{2}$ & $F$ & Prob $>F$ \\
\hline $1 \mathrm{a}$ & 8.16 & 102.7 & -0.84 & 2.43 & 0.9972 & 1986.84 & $<0.0001$ \\
\hline $1 b$ & 5.48 & 100.62 & -0.77 & 3.19 & 0.9949 & 895.97 & $<0.0001$ \\
\hline 1c & 11.67 & 103.61 & -0.83 & 2.45 & 0.9911 & 680.87 & $<0.0001$ \\
\hline 1d & 11.13 & 106.39 & -0.69 & 2.06 & 0.9849 & 371.58 & $<0.0001$ \\
\hline $2 a$ & 15.85 & 102.11 & -0.81 & 2.48 & 0.9831 & 394.85 & $<0.0001$ \\
\hline $2 b$ & 10.00 & 118.30 & -0.68 & 1.14 & 0.9772 & 337.11 & $<0.0001$ \\
\hline $2 \mathrm{c}$ & 23.60 & 103.21 & -0.93 & 2.14 & 0.9904 & 1037.18 & $<0.0001$ \\
\hline $2 d$ & 15.92 & 103.73 & -0.90 & 2.18 & 0.9884 & 655.15 & $<0.0001$ \\
\hline $3 \mathbf{a}$ & 15.59 & 100.91 & -0.79 & 2.69 & 0.9940 & 1070.26 & $<0.0001$ \\
\hline $3 \mathbf{b}$ & 9.53 & 116.12 & -0.66 & 1.27 & 0.9760 & 283.56 & $<0.0001$ \\
\hline $3 \mathrm{c}$ & 20.38 & 103.98 & -0.93 & 1.86 & 0.9931 & 1403.44 & $<0.0001$ \\
\hline 3d & 14.57 & 103.47 & -0.81 & 2.34 & 0.9929 & 920.38 & $<0.0001$ \\
\hline
\end{tabular}

$\mathrm{y}$ : inhibition percentage; A1: bottom asymptote; A2: top asymptote; LOGx0: center; $\mathrm{x}: \log _{10}$ [concentration]; p: hill slope; $\mathrm{r}^{2}$ : coefficient of determination; $F$ : value obtained by the $F$-test; Prob $>F=$ probability value ( $p$ model, $F<0.0001$ ).

groups (a-d), the compounds with the butyl group (c) showed the best results within each series (1a-d, 2a-d and 3a-d), considering both the $\mathrm{IC}_{50}$ and $\mathrm{IC}_{90}$ values for H. vastatrix (Table 6). The same trend was observed for P. pachyrhizi, with one exception within the group 1a-d. Membrane affinity follows a nonlinear behavior, usually increasing with the chain length up to a threshold, beyond which lengthening of the chain results in a reduction on the biological activity. ${ }^{29}$ The better performance of the compounds bearing the butyl group (c) points to an optimum chain length of ca. four carbon atoms for the inhibitory activity here studied.

The introduction of fluorine atoms and nitro groups in biologically active molecules is known to affect their 

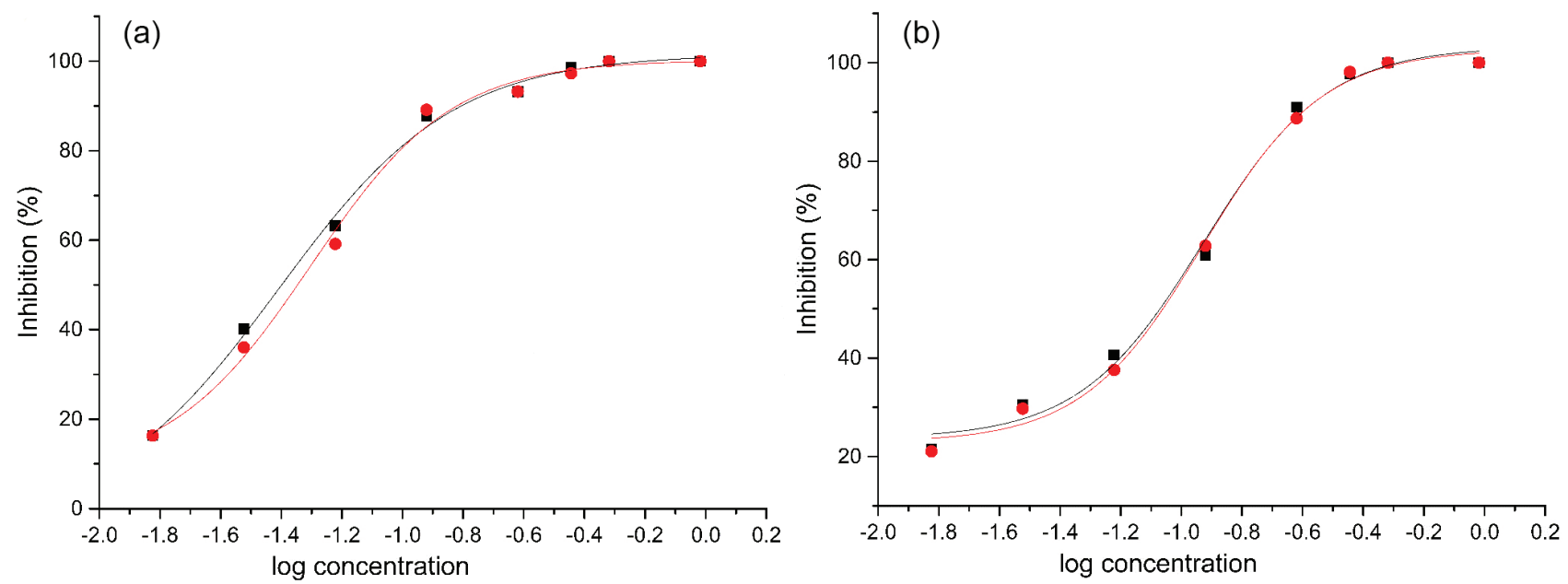

Figure 6. Dose-response curves for the inhibition (in percentage) of $P$. pachyrhizi (a) and $H$. vastatrix (b) spore germination by compound $\mathbf{2 c}$ after $24 \mathrm{~h}$ of incubation at $25^{\circ} \mathrm{C}$, in two separate experiments, with three replicates per treatment in each experiment.

Table 6. Concentrations of the allyldithiocarbimate salts to inhibit $50 \%$ $\left(\mathrm{IC}_{50}\right)$ and $90 \%\left(\mathrm{IC}_{90}\right)$ spore germination of P. pachyrhizi and $H$. vastatrix

\begin{tabular}{lcccc}
\hline & \multicolumn{4}{c}{ Fungi } \\
\cline { 2 - 5 } Compound & \multicolumn{2}{c}{ P. pachyrhizi } & \multicolumn{2}{c}{ H. vastatrix } \\
\cline { 2 - 5 } & $\begin{array}{c}\mathrm{IC}_{50} / \\
\left(\mu \mathrm{mol} \mathrm{L}^{-1}\right)\end{array}$ & $\begin{array}{c}\mathrm{IC}_{90} / \\
\left(\mu \mathrm{mol} \mathrm{L}^{-1}\right)\end{array}$ & $\begin{array}{c}\mathrm{IC}_{50} / \\
\left(\mu \mathrm{mol} \mathrm{L}^{-1}\right)\end{array}$ & $\begin{array}{c}\mathrm{IC}_{90} / \\
\left(\mu \mathrm{mol} \mathrm{L}^{-1}\right)\end{array}$ \\
\hline 1a & 85 & 234 & 130 & 308 \\
1b & 67 & 180 & 161 & 322 \\
1c & 26 & 223 & 129 & 302 \\
1d & 30 & 189 & 172 & 443 \\
2a & 69 & 205 & 132 & 320 \\
2b & 47 & 288 & 129 & 514 \\
2c & 40 & 156 & 85 & 252 \\
2d & 59 & 217 & 101 & 271 \\
3a & 60 & 274 & 142 & 334 \\
3b & 59 & 321 & 149 & 533 \\
3c & 49 & 240 & 84 & 275 \\
3d & 49 & 257 & 130 & 323 \\
\hline
\end{tabular}

effectiveness. The presence of such substituents can alter important parameters such as interaction with enzymes, cell membranes and target receptors, or may produce secondary activity reactions, for example due to the enzymatic bioreduction of nitro groups..$^{30-33}$

Therefore, along with the compounds 1a-d (with the unsubstituted phenyl ring), we included the analogues 2a-d and 3a-d in order to evaluate if the introduction of the nitro and trifluoromethyl substituents would affect the activity of the allyldithiocarbimates. The analysis of the results (Table 6) was not straightforward when considering the presence of these groups in the structures. The effect of both nitro (2) and trifluoromethyl (3) substituents on the $\mathrm{IC}_{90}$ values are positive for the inhibition of $H$. vastatrix when considering the analogues with the longer carbon chains (c and $\mathbf{d})$. Nevertheless, these substituents lower the inhibition of $H$. vastatrix germination when the allyldithiocarbimates bear short alkyl groups (a and b). The results for P. pachyrhizi were even more complex with respect to the influence of the nitro group, while the trifluoromethyl group clearly diminishes the performance of these new antifungals.

\section{Conclusions}

The reactions between $N$-alkyl-sulfonyldithiocarbimates and allylic bromides are very fast and furnish allyldithiocarbimates in good yields. The X-ray diffraction studies confirmed that the reactions are stereospecific, furnishing exclusively the $Z$ isomers, as the spectroscopic data also indicated.

The twelve new compounds inhibited the spore germination of $P$. pachyrhizi and $H$. vastatrix at very low doses. The activities were higher against $P$. pachyrhizi. It was observed that the alkyl chain with four carbon atoms increases the inhibitory activity in comparison with the homologues with one, two or eight carbon atoms, in most cases. The presence of the trifluorophenyl and nitro groups in the aromatic ring enhanced the activity of the allyldithiocarbimates against $H$. vastatrix for the compounds bearing the longer alkyl chains (c and d), showing opposite results for the shorther chains analogues (a and b). The influence of these groups seemd to be more complex with respect to $P$. pachyrhizi.

The best overall results were achieved in the treatments with compound $\mathbf{2 c}$ (substituent on the aromatic ring = nitro; alkyl group $=$ butyl), which resulted in the lowest $\mathrm{IC}_{90}$ values $(252 \mu \mathrm{mol} \mathrm{L})^{-1}$ for $H$. vastatrix, and $156 \mu \mathrm{mol} \mathrm{L}^{-1}$ 
for P. pachyrhizi). The experiments were repeated, and the results are reproducible. Considering the Tukey's test (Figure 4), the activity of compound $\mathbf{2 c}$ on the germination of $P$. pachyrhizi was superior to the result achieved with the positive control (ZDMC) at $120 \mu \mathrm{mol} \mathrm{L}{ }^{-1}$.

Thus, the studies here described indicated that the allyldithiocarbimate salts are a new group of biologically active substances with potential application for the control of coffee leaf rust and Asian sybean rust diseases. Differently from dithiocarbamates such as ZDMC, the allyldithiocarbimates are metal free and form anionic species. We have shown that changes in the structure of the allylic anions can enhance the antifungal activity of their salts. The good results presented by these salts may be further improved by variations on the cation, what can modulate the salt solubility and the activity of this new class of agrochemicals. Compound $\mathbf{2 c}$ is a target substance for further studies on the development of a new fungicide, especially considering the inhibition of $P$. pachyrhizi germination.

\section{Supplementary Information}

Supplementary crystallographic data for $\mathbf{2 a}$ (CCDC 1559144) can be obtained free of charge from the Cambridge Crystallographic Data Centre via www. ccdc.cam.ac.uk/data_request/cif (or from the Cambridge Crystallographic Data Centre, 12, Union Road, Cambridge CB2 1EZ, UK; fax: +44 1223 336033).

Supplementary data are available free of charge at http://jbcs.sbq.org.br as PDF file.

\section{Acknowledgments}

We are grateful to the Fundação de Amparo à Pesquisa do Estado de Minas Gerais (FAPEMIG, grant APQ-02382-17), Conselho Nacional de Desenvolvimento Científico e Tecnológico (CNPq) and Coordenação de Aperfeiçoamento de Pessoal de Nível Superior (CAPES) for financial support and research fellowships. We thank the Núcleo de Análise de Biomoléculas of the Universidade Federal de Viçosa for providing the facilities for HRMS experiments.

\section{References}

1. Hogarth, G.; Prog. Inorg. Chem. 2005, 53, 71.

2. Kanemoto-Kataoka, Y.; Oyama, T. M.; Ishibashi, H.; Oyama,Y.; Chem.-Biol. Interact. 2015, 237, 80.

3. Guo, X.; Zhou, S.; Chen, Y.; Chen, X.; Liu, J.; Ge, F.; Lian, Q.; Chen, X.; Ge, R. S.; Toxicol. Sci. 2017, 160, 329.
4. Alves, L. C.; Rubinger, M. M. M.; Lindemann, R. H.; Perpétuo, G. J.; Janczak, J.; Miranda, L. D. L.; Zambolim, L.; Oliveira, M. R. L.; J. Inorg. Biochem. 2009, 103, 1045.

5. Oliveira, A. A.; Oliveira, M. R. L.; Rubinger, M. M. M.; Rezende, D. C.; Zambolim, L.; Quim. Nova 2015, 38, 757.

6. Vidigal, A. E. C.; Rubinger, M. M. M.; Oliveira, M. R. L.; Guilardi, S.; Souza, R. A. C.; Ellena, J.; Zambolim, L.; J. Mol. Struct. 2016, 1114, 21.

7. Bomfim-Filho, L. F. O.; Oliveira, M. R. L.; Miranda, L. D. L.; Vidigal, A. E. C.; Guilardi, S.; Souza, R. A. C.; Ellena, J.; Ardisson, J. D.; Zambolim, L.; Rubinger, M. M. M.; J. Mol. Struct. 2017, 1129, 60.

8. Tavares, E. C.; Rubinger, M. M. M.; Filho, E. V.; Oliveira, M. R. L.; Veloso, D. P.; Ellena, J.; Guilardi, S.; Souza, R. A. C.; Zambolim, L.; J. Mol. Struct. 2016, 1106, 130.

9. Avelino, J.; Cristancho, M.; Georgiou, S.; Imbach, P.; Aguilar, L.; Bornemann, G.; Läderach, P.; Anzueto, F.; Hruska, A. J.; Morales, C.; Food Secur. 2015, 7, 303.

10. Zambolim, L.; Trop. Plant Pathol. 2016, 41, 1.

11. Godoy, C. V.; Seixas, C. D. S.; Soares, R. M.; MarcelinoGuimarães, F. C.; Meyer, M. C.; Costamilan, L. M.; Pesq. Agropec. Bras. 2016, 51, 407.

12. Oliveira, M. R. L.; de Bellis, V. M.; Transition Met. Chem. 1999, 24, 127.

13. Franca, E. F.; Oliveira, M. R. L.; Guilardi, S.; Andrade, R. P.; Lindemann, R. H.; Amim, J.; Ellena, J.; de Bellis, V. M.; Rubinger, M. M. M.; Polyhedron 2006, 25, 2119.

14. Cunha, L. M. G.; Rubinger, M. M. M.; Sabino, J. R.; Visconte, L. L. Y.; Oliveira, M. R. L.; Polyhedron 2010, 29, 2278.

15. Cai, J. X.; Zhou, Z. H.; Zhao, G. F.; Tang, C. C.; Org. Lett. 2002, 4, 4723.

16. Ferreira, M.; Fernandes, L.; Sá, M. M.; J. Braz. Chem. Soc. 2009, 20, 564.

17. Santhoshi, A.; Mahendar, B.; Mattapally, S.; Sadhu, P. S.; Banerjee, S. K.; Rao, V. J.; Bioorg. Med. Chem. Lett. 2014, 24, 1952.

18. Bruker; APEX2; Bruker AXS Inc., Madison, WI, USA, 2004. 19. Bruker; SAINT; Bruker AXS Inc., Madison, WI, USA, 2007.

20. Bruker; SADABS; Bruker AXS Inc., Madison, WI, USA, 2002.

21. Sheldrick, G. M.; Acta Crystallogr., Sect. A 2008, 64, 112.

22. Sheldrick, G. M.; Acta Crystallogr., Sect. C 2015, 71, 3.

23. Farrugia, L.; J. Appl. Crystallogr. 2012, 45, 849.

24. Macrae, F.; Edgington, P. R.; McCabe, P.; Pidcock, E.; Shields, G. P.; Taylor, R.; Towler, M.; Van de Streek, J.; J. Appl. Crystallogr. 2006, 39, 453.

25. Sebaugh, J. L.; Pharm. Stat. 2011, 10, 128.

26. Vidigal, A. E. C.; Rubinger, M. M. M.; Queiroz, L. F.; Silva, L. F.; Zambolim, L.; Guilardi, S.; Souza, R. A. C.; Ellena, J.; Wetler, E. B.; Oliveira, M. R. L.; Inorg. Chim. Acta 2019, 486, 724.

27. Allen, F. H.; Kennard, O.; Watson, D. G.; Brammer, L.; Orpen, A. G.; Taylor, R.; J. Chem. Soc., Perkin Trans. 2 1987, 2, S1. 
28. Alves, L. C.; Rubinger, M. M. M.; Tavares, E. C.; Janczak, J.; Pacheco, E. B. A. V.; Visconte, L. L. Y.; Oliveira, M. R. L.; J. Mol. Struct. 2013, 1048, 244.

29. Durand, E.; Jacob, R. F.; Sherratt, S.; Lecomte, J.; Baréa, B.; Villeneuve, P.; Mason, R. P.; Eur. J. Lipid Sci. Technol. 2017, 118, 1600397.

30. Theodoridis, G.; Adv. Fluorine Sci. 2006, 2, 121.

31. Goulart, M. O. F.; Souza, A. A. S.; Abreu, F. C.; Paula, F. S.; Sales, E. M.; Almeida, W. P.; Buriez, O.; Amatore, C.; J. Electrochem. Soc. 2007, 154, 121.
32. de Paiva, Y. G.; de Souza, A. A.; Lima-Junior, C. G.; Silva, F. P. L.; Filho, E. B. A.; de Vasconcelos, C. C.; de Abreu, F. C.; Goulart, M. O. F.; Vasconcellos, M. L. A. A.; J. Braz. Chem. Soc. 2012, 23, 894.

33. Jeschke, P.; Pest Manage. Sci. 2017, 73, 1053.

Submitted: July 10, 2019

Published online: October 3, 2019 